\title{
Sinc collocation method for the approximate solutions of Bratu-type equations with conformable derivative
}

\author{
Sertan Alkan \\ Iskenderun Technical University, 31200, Iskenderun, Hatay, Turkey
}

Received: 28 October 2019, Accepted: 23 December 2019

Published online: 26 December 2019.

\begin{abstract}
In this study, Sinc-collocation method is introduced to obtain an approximate solution of fractional Bratu-type equations with boundary conditions. Conformable derivative is considered for the fractional derivatives. Two numerical examples are approximately solved and absolute errors are presented in tables. The results showed that the method is a very efficient to obtain approximate solutions of fractional Bratu-type equations with conformable derivative.
\end{abstract}

Keywords: Sinc collocation method, fractional Bratu-type equations, conformable derivative.

\section{Introduction}

Sinc methods have been recognized as powerful tools for handling problems encountered in scientific and engineering applications. There are several advantages to use approximations based on Sinc methods. Firstly, they are well-established that they are characterized by exponentially decaying errors. Secondly, they are easily implemented and give good accuracy for problems with singularities. Finally, these kinds of approximation give both an effective and quickly convergent scheme for solving the problem and overreaches the instability problems that one mostly confronts in some difference methods. In particular Sinc collocation method can be summarized as follows: The solution function is expanded to a finite series in terms of composite translated sinc functions and some unknown coefficients. These unknown coefficients are determined by Sinc collocation method. For detailed information on Sinc-collocation method, we refer the reader the papers $[1,2]$.

Khalil et al.[3] introduced a new definition of fractional derivative called the conformable fractional derivative. For a detailed overview of the conformable fractional derivative and applications, we refer the reader to $[4,5]$ and references therein.

Particularly, in this study, we apply the sinc-collocation method to the one-dimensional fractional planar Bratu-type problem in the following form

$$
y^{(\alpha)}(x)+\mu e^{y(x)}=0 ; y(0)=0, y(1)=0
$$

where $y^{(\alpha)}$ is the conformable fractional derivative for $1<\alpha \leq 2$.

Approximate solutions of the equation (1) based on Riemann-Liouville and Caputo derivatives has been studied in several articles with various numerical methods. However, in this paper, we focus on the Sinc collocation method to obtain the approximate solution of the equation (1) based on the conformable fractional derivative.

* Corresponding author e-mail: sertan.alkan@iste.edu.tr 
The paper organized as follows. In section 2, we have given some definition and theorems for conformable fractional calculus. In section 3, Sinc-collocation method is presented. In section 4, error estimate for the solution is given. In section 5 , two test problems have been given to show the abilities of the methods. Finally, in section 6 , we have completed the paper with a conclusion.

\section{Preliminaries}

Definition 1. Let $\alpha \in(n, n+1]$, and $f$ be an $n$-differentiable function at $t$, where $t>0$. Then the conformable fractional derivative of $f$ of order $\alpha$ is defined as

$$
T_{\alpha}(f)(t)=\lim _{\varepsilon \rightarrow 0} \frac{f^{(\lceil\alpha\rceil-1)}\left(t+\varepsilon t^{(\lceil\alpha\rceil-\alpha)}\right)-f^{(\lceil\alpha\rceil-1)}(t)}{\varepsilon}
$$

where $\lceil\alpha\rceil$ is the smallest integer greater than or equal to $\alpha$.

Remark.As a consequence of Definition1, one can easily show that

$$
T_{\alpha}(f)(t)=t^{(\lceil\alpha\rceil-\alpha)} f^{\lceil\alpha\rceil}(t)
$$

where $\alpha \in(n, n+1]$, and $f$ is $(n+1)$ differentiable at $t>0$.

Theorem 1. Let $\alpha \in(n, n+1]$ and $f ; g$ be $\alpha$-differentiable at a point $t>0$.Then

(1) $T_{\alpha}(a f+b g)=a T_{\alpha}(f)+b T_{\alpha}(g)$, for all $a, b \in \mathbb{R}$.

(2) $T_{\alpha}\left(t^{p}\right)=p t^{p-\alpha}$, for all $p \in \mathbb{R}$

(3) $T_{\alpha}(\lambda)=0$, for all constant functions $f(t)=\lambda$.

(4) $T_{\alpha}(f g)=f T_{\alpha}(g)+g T_{\alpha}(f)$.

(5) $T_{\alpha}\left(\frac{f}{g}\right)=\frac{g T_{\alpha}(f)+f T_{\alpha}(g)}{g^{2}}$.

Definition 2. The Sinc function is defined on the whole real line $-\infty<x<\infty$ by

$$
\operatorname{sinc}(x)= \begin{cases}\frac{\sin (\pi x)}{\pi x}, & x \neq 0 \\ 1, & x=0\end{cases}
$$

Definition 3. For $h>0$ and $k=0, \pm 1, \pm 2, \ldots$ the translated sinc function with space node are given by:

$$
S(k, h)(x)=\operatorname{sinc}\left(\frac{x-k h}{h}\right)= \begin{cases}\frac{\sin \left(\pi \frac{x-k h}{h}\right)}{\pi \frac{x-k h}{h}}, & x \neq k h \\ 1, & x=k h\end{cases}
$$

To construct approximation on the interval $(a, b)$ the conformal map

$$
\phi(z)=\ln \left(\frac{z-a}{b-z}\right)
$$

is employed. The basis functions on the interval $(a, b)$ are derived from the composite translated sinc functions

$$
S_{k}(z)=S(k, h)(z) \circ \phi(z)=\operatorname{sinc}\left(\frac{\phi(z)-k h}{h}\right) .
$$

The inverse map of $w=\phi(z)$ is

$$
z=\phi^{-1}(w)=\frac{a+b e^{w}}{1+e^{w}}
$$


The sinc grid points $z_{k} \in(a, b)$ will be denoted by $x_{k}$ because they are real. For the evenly spaced nodes $\{k h\}_{k=-\infty}^{\infty}$ on the real line, the image which corresponds to these nodes is denoted by

$$
x_{k}=\phi^{-1}(k h)=\frac{a+b e^{k h}}{1+e^{k h}}, \quad k=0, \pm 1, \pm 2, \ldots
$$

Definition 4. An open set $S \subseteq \mathbb{C}$ is called connected if it cannot be written as the union of two disjoint open sets $A$ and $B$ such that both $A$ and $B$ intersect $S$. An open set $S \subseteq \mathbb{C}$ is called simply connected if $\overline{\mathbb{C}} \backslash S$, where $\overline{\mathbb{C}}$ is the extended complex plane denoted $\mathbb{C} \cup\{\infty\}$, is connected.

Definition 5. Let $D_{E}$ be a simply connected domain in the complex plane $\mathbb{C}$, and let $\partial D_{E}$ denote the boundary of $D_{E}$. Let $a, b$ be points on $\partial D_{E}$ and $\phi$ be a conformal map $D_{E}$ onto $D_{S}$ such that $\phi(a)=-\infty$ and $\phi(b)=\infty$. If the inverse map of $\phi$ is denoted by $\varphi$, define

$$
\Gamma=\left\{\phi^{-1}(u) \in D_{E}:-\infty<u<\infty\right\}
$$

and $z_{k}=\varphi(k h), k=0, \pm 1, \pm 2, \ldots$

Definition 6. Let $B\left(D_{E}\right)$ be the class of functions $F$ that are analytic in $D_{E}$ and satisfy

$$
\int_{\psi(L+u)}|F(z)| d z \rightarrow, a s u=\mp \infty
$$

where

$$
L=\left\{i y:|y|<d \leq \frac{\pi}{2}\right\}
$$

and those on the boundary of $D_{E}$ satisfy

$$
T(F)=\int_{\partial D_{E}}|F(z) d z|<\infty
$$

Theorem 2. Let $\Gamma$ be $(0,1), F \in B\left(D_{E}\right)$, then for $h>0$ sufficiently small,

$$
\int_{\Gamma} F(z) d z-h \sum_{j=-\infty}^{\infty} \frac{F\left(z_{j}\right)}{\phi^{\prime}\left(z_{j}\right)}=\frac{i}{2} \int_{\partial D} \frac{F(z) k(\phi, h)(z)}{\sin (\pi \phi(z) / h)} d z \equiv I_{F}
$$

where

$$
|k(\phi, h)|_{z \in \partial D}=\left|e^{\left[\frac{i \pi \phi(z)}{h} \operatorname{sgn}(\operatorname{Im} \phi(z))\right]}\right|_{z \in \partial D}=e^{\frac{-\pi d}{h}}
$$

For the sinc methods, the infinite quadrature rule must be truncated to a finite sum. The following theorem indicates the conditions under which an exponential convergence results.

Theorem 3. If there exist positive constants $\alpha, \beta$ and $C$ such that

$$
\left|\frac{F(x)}{\phi^{\prime}(x)}\right| \leq C \begin{cases}e^{-\alpha|\phi(x)|}, & x \in \psi((-\infty, \infty)) \\ e^{-\beta|\phi(x)|}, & x \in \psi((0, \infty)) .\end{cases}
$$

then the error bound for the quadrature rule (3) is

$$
\left|\int_{\Gamma} F(x) d x-h \sum_{j=-M}^{N} \frac{F\left(x_{j}\right)}{\phi^{\prime}\left(x_{j}\right)}\right| \leq C\left(\frac{e^{-\alpha M h}}{\alpha}+\frac{e^{-\beta N h}}{\beta}\right)+\left|I_{F}\right|
$$


The infinite sum in (3) is truncated with the use of (4) to arrive at the inequality (5). Making the selections

$$
h=\sqrt{\frac{\pi d}{\alpha M}}
$$

and

$$
N \equiv\left[\left\lfloor\frac{\alpha M}{\beta}+1\right\rfloor\right]
$$

where $[\lfloor\rfloor$.$] is an integer part of the statement and M$ is the integer value which specifies the grid size, then

$$
\int_{\Gamma} F(x) d x=h \sum_{j=-M}^{N} \frac{F\left(x_{j}\right)}{\phi^{\prime}\left(x_{j}\right)}+O\left(e^{-(\pi \alpha d M)^{1 / 2}}\right) .
$$

Lemma 1. [6] Let $\phi$ be the conformal one-to-one mapping of the simply connected domain $D_{E}$ onto $D_{S}$. Then

$\delta_{j k}^{(0)}=\left.[S(j, h) o \phi(x)]\right|_{x=x_{k}} \begin{cases}1, & j=k \\ 0, & j \neq k\end{cases}$

$\delta_{j k}^{(1)}=\left.h \frac{d}{d \phi}[S(j, h) o \phi(x)]\right|_{x=x_{k}} \begin{cases}0, & j=k \\ \frac{(-1)^{k-j}}{k-j}, & j \neq k\end{cases}$

$\delta_{j k}^{(2)}=\left.h^{2} \frac{d^{2}}{d \phi^{2}}[S(j, h) o \phi(x)]\right|_{x=x_{k}} \begin{cases}-\frac{\pi^{2}}{3}, & j=k \\ \frac{-2(-1)^{k-j}}{(k-j)^{2}}, & j \neq k\end{cases}$

\section{The sinc-collocation method}

Let us assume an approximate solution for $y(x)$ in Eq.(1) by finite expansion of sinc basis functions for as follows;

$$
y_{n}(x)=\sum_{k=-M}^{N} c_{k} S_{k}(x), \quad n=M+N+1
$$

where $S_{k}(x)$ is the function $S(k, h) \circ \phi(x)$. Here, the unknown coefficients $c_{k}$ in (7) are determined by sinc-collocation method via the following theorems.

Theorem 4. [7] The conformable fractional derivative of order $\alpha$ of $y_{n}(x)$ for $1<\alpha \leq 2$ is given by

$$
y_{n}^{(\alpha)}(x)=\sum_{k=-M}^{N} c_{k} x^{2-\alpha}\left(\phi^{\prime \prime}(x) \frac{d}{d \phi} S_{k}(x)+\left(\phi^{\prime}(x)\right)^{2} \frac{d^{2}}{d \phi^{2}} S_{k}(x)\right)
$$

Proof. We may write the conformable fractional derivative of order $\alpha$ of $y_{n}(x)$ in (7) as

$$
y_{n}^{(\alpha)}(x)=\sum_{k=-M}^{N} c_{k} S_{k}^{(\alpha)}(x)
$$

By using Remark 1, we have

$$
S_{k}^{(\alpha)}(x)=x^{2-\alpha} S_{k}^{\prime \prime}(x)
$$

Then by Eq.(9), we get the desired result

$$
y_{n}^{(\alpha)}(x)=\sum_{k=-M}^{N} c_{k} x^{2-\alpha}\left(\phi^{\prime \prime}(x) \frac{d}{d \phi} S_{k}(x)+\left(\phi^{\prime}(x)\right)^{2} \frac{d^{2}}{d \phi^{2}} S_{k}(x)\right)
$$


Replacing each term of (1) with the approximation given in (7)-(8), multiplying the resulting equation by $\left\{\left(1 / \phi^{\prime}\right)^{2}\right\}$, then setting $x=x_{j}$, we obtain the following theorem.

Theorem 5. If the assumed approximate solution of boundary value problem (1) is (7), then the discrete sinc-collocation system for the determination of the unknown coefficients $\left\{c_{k}\right\}_{k=-M}^{N}$ is given by

$$
\sum_{k=-M}^{N}\left[c_{k}\left\{\frac{1}{h^{2}} \delta_{j k}^{(2)}\left[\phi^{\prime}\left(x_{j}\right)\right]^{2}-\frac{1}{h} \delta_{j k}^{(1)} \phi^{\prime \prime}\left(x_{j}\right)\right\}\left(x_{j}\right)^{2-\alpha}\right]+\mu e^{c_{j}}=0 \quad j=-M, \ldots, N
$$

\section{Error estimate for the Solution}

In this section, we summarize an error estimation given in [8] to check the accuracy of the proposed method. Let $y_{n}(x)$ is an approximate solution to Equation (1). Assume that $y_{n}(x)$ and its conformable derivative are substituted into Equation (1), then the obtained equations should satisfy approximately, in short,

$$
E\left(x_{r}\right)=\left|y_{n}^{(\alpha)}\left(x_{r}\right)+e^{y_{n}\left(x_{r}\right)}\right| \cong 0
$$

for $x=x_{r}, x_{r} \in[0,1]$.

\section{Computational examples}

Here we give two numerical examples to illustrate the accuracy of the proposed method.

\subsection{Example}

Consider fractional planar Bratu-type problem in the following form

$$
y^{(\alpha)}(x)+e^{y(x)}=0
$$

subject to the homogeneous boundary conditions

$$
y(0)=y(1)=0
$$

Absolute errors for this problem when $\alpha=1.2$ are given in Table 1. Also, the numerical solutions which are obtained by using the present method for $N=64$ and different values of $\alpha$ are presented in Table 2. In table 3, absolute errors and numerical results for $N=64$ and $\alpha=2$ are given. The graphs of approximate solutions for different values of $\alpha$ are given in Figure 1. Graphs in Figure 1 show that when $\alpha$ approaches to 2, the corresponding solutions of fractional order differential equation approach to the solutions of integer order differential equation.

\subsection{Example}

Consider fractional Bratu-type problem in the following form

$$
y^{(\alpha)}(x)-2 \pi e^{y(x)}=0
$$


Table 1: Absolute errors for Example 1 for different values of $N$ when $\alpha=1.2$

\begin{tabular}{ccccc}
\hline$x$ & $N=16$ & $N=32$ & $N=64$ & $N=128$ \\
\hline \hline 0.0 & 0.0 & 0.0 & 0.0 & 0.0 \\
0.2 & $1.827 \times 10^{-4}$ & $2.713 \times 10^{-6}$ & $7.957 \times 10^{-8}$ & $1.826 \times 10^{-8}$ \\
0.4 & $1.046 \times 10^{-4}$ & $3.146 \times 10^{-7}$ & $1.489 \times 10^{-7}$ & $3.507 \times 10^{-8}$ \\
0.6 & $1.347 \times 10^{-4}$ & $5.984 \times 10^{-7}$ & $1.188 \times 10^{-7}$ & $2.345 \times 10^{-8}$ \\
0.8 & $4.272 \times 10^{-4}$ & $6.251 \times 10^{-6}$ & $2.625 \times 10^{-8}$ & $6.042 \times 10^{-9}$ \\
1.0 & 0.0 & 0.0 & 0.0 & 0.0 \\
\hline
\end{tabular}

Table 2: Absolute errors for Example 1 for $N=64$ and different values of $\alpha$

\begin{tabular}{ccccc}
\hline$x$ & $\alpha=1.2$ & $\alpha=1.4$ & $\alpha=1.6$ & $\alpha=1.8$ \\
\hline \hline 0.0 & 0.0 & 0.0 & 0.0 & 0.0 \\
0.2 & $8.604 \times 10^{-9}$ & $6.970 \times 10^{-9}$ & $6.616 \times 10^{-9}$ & $3.517 \times 10^{-9}$ \\
0.4 & $7.237 \times 10^{-9}$ & $1.090 \times 10^{-8}$ & $1.444 \times 10^{-8}$ & $8.914 \times 10^{-10}$ \\
0.6 & $2.248 \times 10^{-8}$ & $1.504 \times 10^{-8}$ & $1.519 \times 10^{-8}$ & $9.279 \times 10^{-9}$ \\
0.8 & $3.645 \times 10^{-9}$ & $4.043 \times 10^{-9}$ & $5.009 \times 10^{-9}$ & $3.665 \times 10^{-9}$ \\
1.0 & 0.0 & 0.0 & 0.0 & 0.0 \\
\hline
\end{tabular}

Table 3: Absolute errors and numerical results for Example 1 for $N=64$ and $\alpha=2$

\begin{tabular}{ccc}
\hline$x$ & Numerical results & Absolute Errors \\
\hline \hline 0.0 & 0.0 & 0.0 \\
0.2 & 0.08918 & $9.110 \times 10^{-9}$ \\
0.4 & 0.13479 & $9.578 \times 10^{-9}$ \\
0.6 & 0.13479 & $9.577 \times 10^{-9}$ \\
0.8 & 0.08918 & $9.110 \times 10^{-9}$ \\
1.0 & 0.0 & 0.0 \\
\hline
\end{tabular}

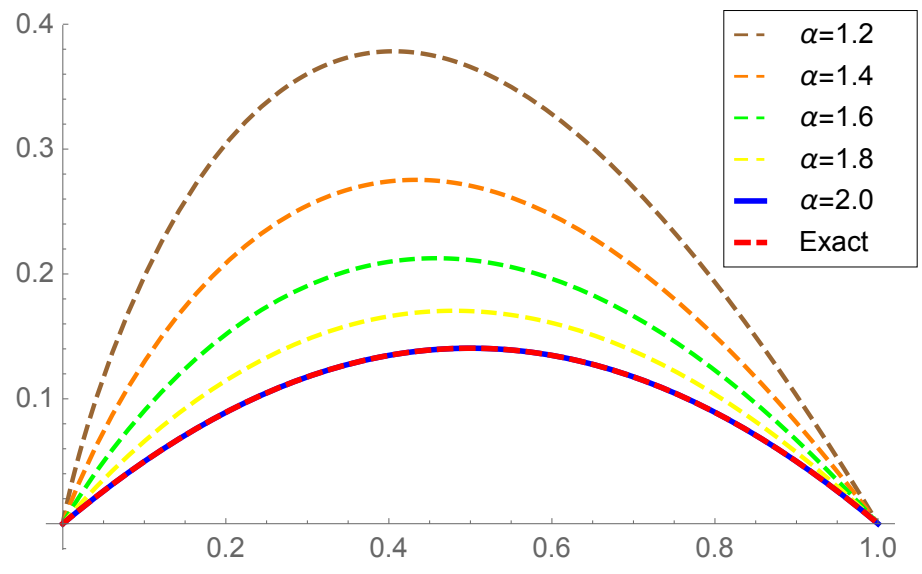

Fig. 1: Graphs of exact and approximate solutions for different values of $\alpha$ for Example 1 
Table 4: Absolute errors for Example 2 for different values of $N$ when $\alpha=1.8$

\begin{tabular}{ccccc}
\hline$x$ & $N=16$ & $N=32$ & $N=64$ & $N=128$ \\
\hline \hline 0.0 & 0.0 & 0.0 & 0.0 & 0.0 \\
0.2 & $1.718 \times 10^{-4}$ & $3.834 \times 10^{-6}$ & $3.404 \times 10^{-10}$ & $3.399 \times 10^{-10}$ \\
0.4 & $5.779 \times 10^{-5}$ & $1.786 \times 10^{-7}$ & $2.172 \times 10^{-9}$ & $8.427 \times 10^{-10}$ \\
0.6 & $5.537 \times 10^{-5}$ & $1.744 \times 10^{-7}$ & $4.056 \times 10^{-9}$ & $8.027 \times 10^{-10}$ \\
0.8 & $1.352 \times 10^{-4}$ & $3.155 \times 10^{-6}$ & $3.858 \times 10^{-9}$ & $3.530 \times 10^{-10}$ \\
1.0 & 0.0 & 0.0 & 0.0 & 0.0 \\
\hline
\end{tabular}

Table 5: Absolute errors for Example 2 for $N=64$ and different values of $\alpha$

\begin{tabular}{ccccc}
\hline$x$ & $\alpha=1.2$ & $\alpha=1.4$ & $\alpha=1.6$ & $\alpha=1.8$ \\
\hline \hline 0.0 & 0.0 & 0.0 & 0.0 & 0.0 \\
0.2 & $4.885 \times 10^{-8}$ & $1.384 \times 10^{-8}$ & $7.981 \times 10^{-9}$ & $1.559 \times 10^{-9}$ \\
0.4 & $4.445 \times 10^{-8}$ & $1.391 \times 10^{-8}$ & $9.746 \times 10^{-9}$ & $6.606 \times 10^{-10}$ \\
0.6 & $1.285 \times 10^{-7}$ & $4.946 \times 10^{-8}$ & $2.401 \times 10^{-8}$ & $6.554 \times 10^{-9}$ \\
0.8 & $2.680 \times 10^{-8}$ & $1.437 \times 10^{-8}$ & $9.076 \times 10^{-9}$ & $4.754 \times 10^{-9}$ \\
1.0 & 0.0 & 0.0 & 0.0 & 0.0 \\
\hline
\end{tabular}

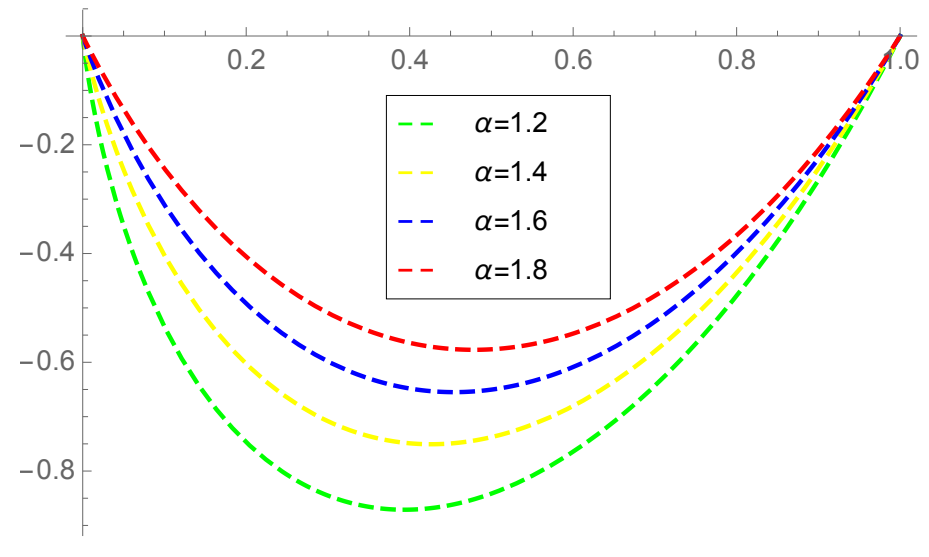

Fig. 2: Graphs of approximate solutions for different values of $\alpha$ for Example 2

subject to the homogeneous boundary conditions

$$
y(0)=y(1)=0
$$

Absolute errors for this problem when $\alpha=1.8$ are given in Table 4. Also, the numerical solutions which are obtained by using the present method for $N=64$ and different values of $\alpha$ are presented in Table 5. The graphs of approximate solutions for different values of $\alpha$ are presented in Figure 2. 


\section{Conclusion}

In this paper, sinc-collocation method is applied to obtain an approximate solution of fractional Bratu-type equations with boundary conditions. Conformable derivative is considered for the fractional derivatives. Two test problems are approximately solved and absolute errors are presented. The numerical results showed that the method is a powerful tool for solving fractional Bratu-type equations, approximately.

\section{Competing interests}

The authors declare that they have no competing interests.

\section{Authors' contributions}

All authors have contributed to all parts of the article. All authors read and approved the final manuscript.

\section{Acknowledgement}

This paper is an extended and revised version of a paper presented at International conference on computational methods in applied sciences with the same title.

\section{References}

[1] Saadatmandi, A., and Dehghan, M., The use of Sinc-collocation method for solving multi-point boundary value problems. Communications in Nonlinear Science and Numerical Simulation, 17(2), (2012): 593-601.

[2] Parand, K., and Pirkhedri, A., Sinc-collocation method for solving astrophysics equations. New Astronomy, 15(6), (2010): 533537.

[3] Khalil, R., Al Horani, M., Yousef, A., and Sababheh, M., A new definition of fractional derivative. Journal of Computational and Applied Mathematics, 264, (2014): 65-70.

[4] Abdeljawad, T., On conformable fractional calculus. Journal of computational and Applied Mathematics, 279, (2015): 57-66.

[5] Benkhettou, N., Hassani, S., and Torres, D. F., A conformable fractional calculus on arbitrary time scales. Journal of King Saud University-Science, 28(1), (2016): 93-98.

[6] Hatipoglu, V. F., Alkan, S., Secer, A., An efficient scheme for solving a system of fractional differential equations with boundary conditions. Advances in Difference Equations, (2017), 2017(1), 204.

[7] Bayram, M., Hatipoglu, V. F., Alkan, S., Das, S. E., A solution method for integro-differential equations of conformable fractional derivative. Thermal Science, (2017), 22(1), S7-S14.

[8] Secer, A., Ozdemir, N. and Bayram, M., A Hermite Polynomial Approach for Solving the SIR Model of Epidemics. Mathematics, (2018), 6(12):305.

[9] Alkan, S., Sinc collocation method for the approximate solutions of fractional Bratu-type equations, International conference on computational methods in applied sciences, July 12-19, 2019, Istanbul, Turkey. 